\title{
Polysèmes
}

Revue d'études intertextuelles et intermédiales

\section{L'écriture du jaune et les traversées de lumière dans The Winter Sleep of Captain Lemass de Harry Clifton}

\section{Cathy Roche-Liger}

\section{OpenEdition}

Journals

Édition électronique

URL : http://journals.openedition.org/polysemes/1467

DOI : $10.4000 /$ polysemes. 1467

ISSN : 2496-4212

Éditeur

SAIT

Référence électronique

Cathy Roche-Liger, «L'écriture du jaune et les traversées de lumière dans The Winter Sleep of Captain Lemass de Harry Clifton », Polysèmes [En ligne], 16 | 2016, mis en ligne le 15 novembre 2016, consulté le 03 mai 2019. URL : http://journals.openedition.org/polysemes/1467 ; DOI : 10.4000/ polysemes. 1467

Ce document a été généré automatiquement le 3 mai 2019.

Polysèmes 


\title{
L'écriture du jaune et les traversées de lumière dans The Winter Sleep of Captain Lemass de Harry Clifton
}

\author{
Cathy Roche-Liger
}

1 Le poète irlandais contemporain Harry Clifton, membre de Aosdána ${ }^{1}$, a occupé la chaire de Ireland Professor of Poetry de 2010 à 2013. Né à Dublin en 1952, il a longtemps vécu à l'étranger, notamment en Afrique, en Asie et en Europe, avant de revenir en Irlande en 2004. Ses voyages ont une influence majeure sur sa poésie, où le thème du déplacement est central. Dans « Coming Home » (2006), où il retrace ses divers retours en Irlande, ainsi que ses départs («the need to leave »), il mêle histoire personnelle et collective (7-16). Dans cet article, dont le sous-titre est «Home to nowhere " et où le péché originel ( Original $\sin »)$ traduit le choix de ses parents de revenir s'installer en République d'Irlande en 1950 (7, appelé aussi «grey republic», 9), il se définit comme un réfugié cosmopolite souffrant d'un sentiment d'aliénation («identity problems », « others like me in the largest tribe of all, the tribe of the displaced», "cosmopolitan», 9, 14). Puisqu'aucun lieu ne peut être une patrie ou un chez-soi, il décrit les mouvements d'exil et de retour au point de départ non comme des traversées permettant d'arriver à bon port, mais comme une errance qui peut devenir une forme de stagnation («I too have wandered the hills of Ireland »; « Exiles like myself who never moved an inch »; « not [...] a going home ", 15-16). Fintan O'Toole, dans un article où il souligne l'importance de Secular Eden (2007), met en relief la notion de mouvement au sein de l'œuvre du poète : «an Irish voice that is utterly contemporary in its restless movement through time and space » (O'Toole 2008). Dans son compte rendu du recueil de 2012 intitulé The Winter Sleep of Captain Lemass (ci-après WS), Benjamin Keatinge écrit: " his poems deliberately cultivate a kind of detached and exilic awareness, an "original homelessness" as it is termed in "Oweniny, Upper Reaches" which allows Clifton to navigate what he has called "the big muddy river of universal history" » (101). Dans « Coming Home», Clifton met en avant ce sentiment de détachement tout en le précisant : « One travelled, not just to gain detachment, but to grow up» (14). Il ajoute: «As Eliot says, here and there cease to matter as one grows older. Only the common human fate remains. And the city named in 
a poem [...] is incidental, no more than a prism through which a universal experience refracts itself» (15). La dialectique de l'ici et de l'ailleurs ("here» / "there», " elsewhere ", « faraway ») est transcendée et fait place à un « destin humain commun " (14-15) ${ }^{2}$. Dans WS, Clifton offre une réflexion sur le passé, le présent, le futur, l'avoir-été, l'être et le devenir sous le signe des traversées, qu'elles soient intertextuelles, intermédiales, temporelles ou géographiques. Si le titre et la référence explicite à l'hibernation suggèrent une immobilité, une forme de suspension; si l'enfer de l'histoire peut s'apparenter à un hiver infini, à une forme de congélation ou de pétrification ${ }^{3}$, le poète ne présente pas pour autant un long sommeil. Il n'y a pas seulement stase, stagnation, répétition, mais aussi déplacement, transfert, devenir. L'impression de mouvement est suggérée dès que nous posons les yeux sur l'ouvrage ou que nous en lisons la table des matières: la photographie de couverture représente des personnages traversant une étendue blanche, et les trois parties qui composent le recueil s'intitulent respectivement "Vingt-six Comtés», "Six Comtés» et "Ailleurs» («Twenty-Six Counties", "Six Counties ", "Elsewhere ", WS, 9, 47, 75). Ainsi, l'Irlande et le monde comme terres fracturées et terres d'exil s'écrivent à travers les mouvements divergents et incessants des individus qui la composent, en dépit de la route enneigée et sans futur que semble être l'histoire ${ }^{4}$. Si ces déplacements peuvent se transformer en une errance qui ne mène nulle part, l'espace poétique fait apparaître des lieux où l'on peut certes se perdre, mais aussi se trouver, une « Carte du Devenir » :

Land, sky and water

Meet out there at a point. Or call it the soul

On Sunday afternoon, at large in a space

To be lost in, where no one wishes to be

And everyone, sooner or later,

Finds himself, on the map of his own becoming.

(WS, « The Map of Becoming », 72)

WS apparait comme une quête de soi et de sens, comme l'écriture du passage sur terre et comme un questionnement existentiel. En quoi l'écriture du jaune, les traversées de lumière et les obscurcissements peuvent-ils être perçus comme les symboles de ce cheminement? Comment Clifton crée-t-il ce faisant un dialogue avec d'autres artistes par l'entremise de l'intertextualité qui parcourt l'œuvre ? Les différents sous-textes sont à la fois une forme de fondation qui vient soutenir le questionnement de Clifton et ce que le poète traverse, transperce, déconstruit pour donner à son texte, qui fonctionne comme "un prisme $~_{5}$ les réfractant, un nouvel éclairage, un nouveau mouvement ou cheminement qui aboutit à une nouvelle vision. Nous proposons ici d'étudier cette traversée intertextuelle qui, pour reprendre et transposer la formule de Louis Marin, s'effectue "dans les deux sens ${ }^{6}$ et qui s'étend, se complexifie lorsqu'elle entre en résonance avec des effets d'intratextualité et d'intermédialité, en prenant pour fil conducteur l'écriture du jaune et de la lumière. Nous décrirons d'abord la manière dont, à travers l'expression d'une quête de sens et de direction, le jaune et la lumière se font traversée, percée, signe d'espoir, même fugace, naissance ou renaissance, prenant une dimension spirituelle, quasi-mystique, avant d'analyser ensuite la façon dont cette lumière peut s'affaiblir, s'obscurcir, se dénaturer, voire n'être plus que la trace de la grâce disparue dans un monde déchu. 


\section{L'écriture du jaune et de la lumière : « une prière séculière? »}

Dans WS, Clifton décrit un monde privé de Dieu : «Dieu est Mort » («God is Dead», 26), déclare-t-il dans « Birr ». Ceci fait écho au nihilisme nietzschéen et soulève des questions de perte de sens en l'absence d'ordre divin. Le poème s'ouvre sur une parodie du Prologue de l'Évangile selon Saint Jean : «In the beginning was the Word / Or call it Birr, a little midland town » (25). Dans cette ville du comté d'offaly, au cœur de l'Irlande, dont le nom évoque par ses sonorités le mot «naissance » de manière tronquée («Birr »/« birth»), il n'y a pas de véritable aube. Il n'existe plus que la répétition d'un simulacre de commencement. À Birr, Dieu et le romancier britannique, Anthony Trollope ${ }^{7}$, qui sont associés et mis sur le même plan, n'ont plus d'indications quant au chemin à suivre. C'est un lieu sombre, associé à la couleur noire (" the black heart of the midlands », « the black end of the empire ", 26-27), où la lumière ne peut plus être qualifiée de céleste ; elle est uniquement ténue et artificielle : lueur des lampadaires obscurcie par la pluie ( In the street-lit small hours, the rain sheeting down », 25), ou celle de la lune et des étoiles décrites comme appartenant à un modèle réduit de l'univers (« His Lordship, playing with space and time, / Switches on the moon and stars / of a model universe ", 26). C'est un monde de faux-semblants (prostitution, alcool, rituel de la messe désormais vide de sens) tout aussi factice qu'une page de roman où seul le rêve et l'amour semblent encore faire sens, ou du moins créer l'illusion d'un sens :

[...] the drunken laughter of girls

With a moral age of centuries, who know it all

And do it darkly [...].

Postmaster Trollope, stationed at Birr,

Grows bored, begins a novel.

Rain and turfsmoke.

[...] God is dead -

The locals, of course, still swarm to church,

Sleep justly, die in bed.

And what of ourselves, as we emerge

So sleepily, on the latest page

Where Trollope and God

Have lost the plot? [...] as the market square

Lets up its shutters

One more time, on a makebelieve,

A storybook town, a dream unshattered

The morning after love. (25-26)

C'est dans ce contexte qu'il faut replacer la manière apparemment paradoxale avec laquelle Clifton se définit dans "Little Jerusalem", à savoir comme "un apostat, / Un adorateur des lumières du ciel » : « an apostate, / A worshipper of skylights » (10). Dans ce poème d'ouverture, il se présente également comme un Juif errant ( $\mathrm{A}$ wandering Jew ", 11) au sein d'un monde où les hommes sont tous décrits comme des exilés («Everyone now an Israelite / Far from home », 10). C'est comme si, dans ce poème construit autour d'un questionnement sur la notion de maison, de chez-soi - foyer et/ou terre natale - la malédiction du Juif errant, condamné à errer jusqu'à la fin des temps, était étendue à l'humanité entière. À un niveau intertextuel, cette expression fait 
référence à Ulysses de James Joyce où le personnage de Leopold Bloom est l'équivalent à la fois d'Ulysse et de la figure du Juif errant ${ }^{8}$.

Dans « Little Jerusalem », où Clifton développe les thèmes de l'exil, de la destruction et de la mémoire et où il se refuse même un statut autre que celui d'exilé ( «Let me not be granted entry / To my homeland», 11), l'Irlande est cette fois grise ("And the grey realisation - Ireland», 11). Ce poème entre en résonance avec « New Jerusalem » de Louis MacNeice et les teintes de gris d'un présent morne («greynesses », 23) . Dans le texte de MacNeice, qui s'ouvre sur une forme impérative liant destruction et mémoire («Bulldoze all memories and sanctuaries»), le sentiment d'aliénation vis-à-vis de la nouvelle Londres, parodie de Jérusalem céleste, surgit de l'abolition du passé. Dans «Little Jerusalem ", la syntaxe parataxique ainsi que les changements de tonalités miment le cheminement brisé de la mémoire dans lequel le lecteur est entrâné («the broken trance / Of remembrance ", 12). Ils sont rythmés par la citation tronquée du psaume 137 ("If I forget thee, O Jerusalem »), psaume qui résonne lui aussi avec les thèmes centraux du poème. Ces changements sont également liés aux effets intertextuels. Le premier vers ("If you have to live somewhere », 10) évoque l'anaphore utilisée par T.S. Eliot dans la première partie de "Little Gidding» pour interpeller son lecteur («If you came this way », Eliot 201). Cet écho est renforcé par le choix du titre «Little Jerusalem», qui rappelle "Little Gidding ", un monastère anglican du XVII ${ }^{e}$ siècle, lieu de dévotion où l'on se rend pour chercher un renouveau spirituel, un lieu de pèlerinage. C'est cette même tonalité, que nous pourrions qualifier d'eliotienne, qui se fait entendre à la fin du poème. Au centre, entre les deux citations du psaume $(10,11)$, ce sont les vers de Wordsworth que Clifton convoque, alors que les vers libres se teintent d'un lyrisme sombre, et que le poète choisit pour résidence les ruines des religions (« Let the ruins of religions be my dwellingplace »,WS, 10). C'est la vision positive de la mémoire selon Wordsworth qu'il déconstruit avec en sous-texte « Lines Written a Few Miles Above Tintern Abbey »:

Thy memory be as a dwelling place

For all sweet sounds and harmonies; oh! Then,

If solitude, or fear, or pain, or grief,

Should be thy portion, with what healing thoughts

of tender joy wilt thou remember me [...].

Nor [...] wilt thou then forget [...] that I, so long

A worshipper of Nature, hither came [...].

Nor wilt thou then forget,

That after many wanderings, many years

of absence, these steep woods and lofty cliffs,

And this green pastoral landscape, were to me

More dear, both for themselves and for thy sake.

(49, c'est moi qui souligne)

6 Quand Clifton se présente comme « un adorateur des lumières du ciel » («A worshipper of skylights»), il répond à Wordsworth qui se définit comme "un adorateur de la Nature » («A worshipper of Nature »). De surcroît, les notions d'errance, de pèlerinage et de mémoire sont communes aux deux poèmes. Cependant, dans le texte de Clifton, la poésie n'a plus pour source, comme chez Wordsworth, un souvenir exaltant et heureux (« Preface » to Lyrical Ballads, 199-200 : « emotion recollected in tranquility », « in a state of enjoyment»). La mémoire n'est ni joyeuse ni harmonieuse. Elle est associée à un cheminement brisé («the broken trance of rememberance », WS, 12) qui met en lumière les traces de ce qui n'est plus, et qui amène le poète à percevoir le monde comme les ruines d'une église profanée : «Let the ruins of religions be my dwelling-place / [...] this 
desecrated temple / Everyone shares [...]» $(10-11)^{10}$. Ce lieu est sa destination en tant qu'exilé : «A wandering Jew [....] getting off [...] / To this, the latest station [...] / The ruins of a church / And forty years in the wilderness » (10-12). Il est néanmoins important de souligner que le choix de ce lieu de résidence a une dimension à la fois symbolique et biographique puisque clifton vit dans une église déconsacrée située dans le quartier de Dublin appelé Little Jerusalem.

7 De plus, dans ce temple en ruines, Clifton offre également une vision positive de l'humanité, d'êtres «étrangement éclairés» après l'amour («strangely lit», 11). L'élévation qu'elle représente par rapport à un monde qui s'effondre est préfigurée par le parcours ascendant du poète, gravissant les marches («I climb», 10). Il associe cette perception de l'humanité à une pause dans son cheminement incessant et douloureux, et l'assimile à une "prière séculaire » («A secular prayer?», 11), et ce même si le point d'interrogation qu'il ajoute après cette expression vient projeter une ombre d'incertitude quant à la réalité d'une telle possibilité :

High in the choirloft, light-motes, angels move

On the firmament of a ceiling. Listen -

A white noise of bathwater running, after love,

And voices, at the hour of vespers, smells of food,

The human returning... Horae Canonicae -

Can I not find them beautiful and good,

So strangely lit, in this desecrated temple

Everyone shares, my pause on the stair

A secular prayer? (10-11)

Clifton associe ici le pictural, le sonore et l'olfactif de manière synesthésique. L'effet pictural créé par la vision des anges, qui évoque les plafonds peints des églises, entre en résonance avec l'allusion à Chagall (11), qui vient elle-même renforcer l'image du Juif errant ("wandering Jew », 11). Par le choix de la lumière du soir, celle du soleil couchant, de la fin du jour, il reste dans la thématique du déclin et de la ruine. Or, en parlant de vêpres et d'heure canonique religieuse, il renvoie aussi au sacré, même si ce n'est qu'à une trace du sacré. Cela fait aussi écho au poème «Vespers» de W.H. Auden, qui fait partie d'une série rassemblée sous le titre « Horae Canonicae» (1976, 627-642). Par cette nouvelle traversée intertextuelle, il convoque tout en le déconstruisant un poème qui explore et rejette différentes visions de ce qui mènerait à la félicité, le nouvel Éden de l'Acardien, qui s'oppose à la Nouvelle Jérusalem de l'Utopiste ${ }^{11}$, et où le sang sacrificiel de l'innocent est perçu cependant par l'Arcadien et l'Utopiste, comme le ciment qui fait tenir les murs séculiers: "for without a cement of blood (it must be human, it must be innocent) no secular wall will safely stand» (1976, 637-639). Dans «Little Jerusalem », il n'y a pas d'exploration des manières illusoires d'accéder au bonheur, ni comme chez Auden d'interprétation chrétienne de l'histoire avec une réaffirmation du rôle de la Crucifixion, mais la description d'un monde séculier, postmoderne et post-religieux ${ }^{12}$. Pourtant ici, dans cette petite Jérusalem, après l'acte d'amour, la lumière déclinante qui vient éclairer les êtres se fait lueur d'espoir. L'amour et la lumière ${ }^{13}$ - ou plutôt ce qu'il en reste - sont le ciment qui fait tenir les fondations de cette citadelle en ruines et qui l'empêche de s'écrouler totalement. Si le monde de Clifton est séculier, il porte la trace du sacré, et si dans ce monde Dieu n'est plus, le poète garde une forme de spiritualité, de mysticisme qu'il formalise ici par les deux expressions paradoxales suivantes: "An apostate, a worshipper of skylights", «a secular prayer»(WS, 10-11) ${ }^{14}$. Enfin, par 
l'entremise de ces allusions aux lumières venues du ciel et au firmament du plafond, ce poème entre en résonance avec " The Skylight » de Seamus Heaney (18) où la lumière qui entre par l'ouverture dans le toit de la maison semble avoir une dimension miraculeuse, le pouvoir de guérir et d'effacer les péchés.

Dans "Daffodils ", la contemplation de la lumière est aussi une expérience à la fois séculière et spirituelle qui pourrait être définie comme un mysticisme séculier de l'immanence.

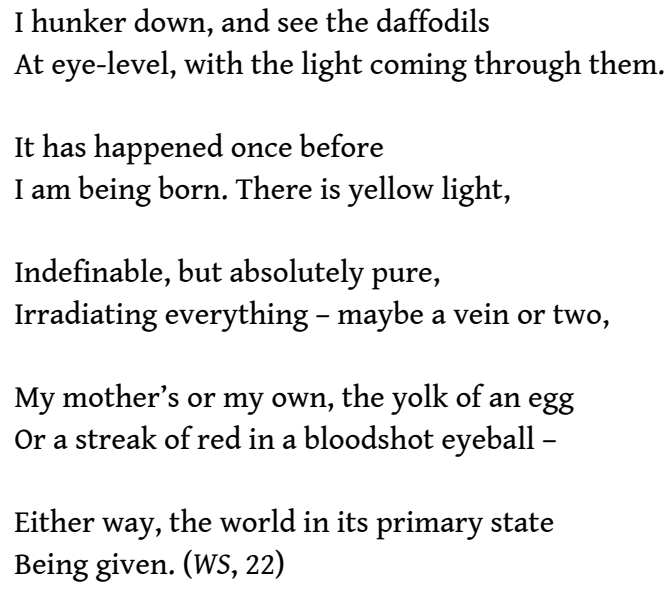

C'est une re-naissance, un recommencement, un nouvel Éden, un "Éden séculier » («a Secular Eden »), pour reprendre le titre du recueil de 2007 de Clifton, que le poète présente ici avec ces jonquilles et ce jaune primaire, pur, puissant. Par l'entremise du titre, il crée un pont avec «I wandered lonely as a cloud» de Wordsworth, aussi connu sous le nom de «Daffodils» (98-99). Or, le poème de Clifton peut être lu comme une réécriture de la version wordsworthienne qui entre en résonance avec «Little Jerusalem » (WS, 10-12) et sa déconstruction postmoderne de «Lines Written a Few Miles Above Tintern Abbey " (44-49). Dans «Daffodils» de Wordsworth, il y a harmonie, ressourcement, et le poète, flottant « tel un nuage », a une position élevée :

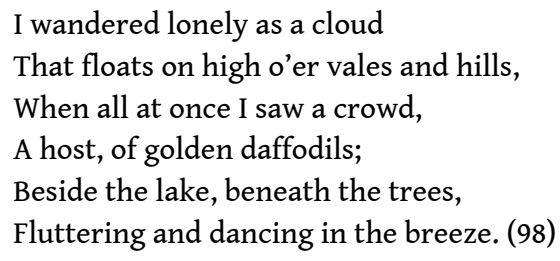

11 Clifton, par le mouvement d'accroupissement, nous ramène vers le sol et introduit d'emblée une notion de gravité. Cette position accroupie, sorte de position fœtale qui annonce sa re-naissance, est aussi un mouvement de régression vers une forme d'innocence temporairement retrouvée. Ceci permet au poète d'être à hauteur d'yeux avec les jonquilles, en harmonie avec la nature, mais d'un autre point de vue, littéralement au ras-du-sol. Il s'ancre ainsi dans une réalité terre à terre. En outre, le jaune décrit par Clifton contraste avec la teinte dorée des jonquilles dans la version romantique, et nous pouvons interpréter ce passage du doré au jaune comme la marque d'un rejet du sacré et la revendication du profane. Cependant, si cette expérience visuelle est de l'ordre du séculier, elle n'en est pas moins signifiante : expérience de sa présence au monde, d'une forme de plénitude dans l'immanence, elle conduit le poète à se redéfinir et à s'associer à la couleur jaune : « Ever afterwards / Yellow is my colour » (WS, 22). C'est l'intensité d'un effet de présence qui est décrit ici comme un phénomène qui se déploie, une re-naissance. Cela correspond à la notion de présence définie par Jean-Luc Nancy 
comme une naissance, quelque chose qui ne peut être saisi comme un état stable ou s'inscrire dans la permanence mais qui s'associe au contraire à un surgissement et à une disparition : « Presence itself is birth, the coming that effaces itself and brings itself back. [...] Joy, jouissance, to come, have the sense of birth: the sense of the inexhaustible imminence of sense " (Nancy 5). Enfin, par cet accroupissement, Clifton prend une posture opposée à celle de Wordsworth, ce qui préfigure le mouvement inverse qui s'opère au fil du poème. Dans la version de Wordsworth, il y a expérience visuelle (la rencontre avec les jonquilles) qui ne fait pas pleinement sens de prime abord, mais qui prend toute sa portée dans la répétition qui s'effectue de manière mémorielle :

I gaz'd - and gaz'd - but little thought

What wealth the show to me had brought:

For oft, when on my couch I lie

In vacant or in pensive mood,

They flash upon that inward eye

Which is the bliss of solitude;

And then my heart with pleasure fills,

And dances with the Daffodils. (98-99, c'est moi qui souligne)

En revanche, dans le poème de Clifton, l'expérience visuelle extrêmement signifiante, qui entraîne une transformation intime immédiate, n'aboutit ensuite qu'à des répétitions appauvries. Les nouvelles occurrences du jaune printanier ne sont que des reflets, des imitations qui ont perdu toute puissance :

Yellow is my colour. And it multiplies

Endlessly. But nothing is the same.

The Spring comes in. Again it is making windows

of itself, to be seen but not seen through. (22)

Elles ne sont plus que des sortes de trompe-l'œil qui ne renvoient plus ni à un état premier et pur ni à aucun au-delà de la sensation. Il n'y a plus ni réelle percée, ni traversée, et l'« Éden séculier » est lui aussi perdu.

À l'instar de ce poème, le jaune et la lumière apparaissent comme des topoï du recueil de Clifton. Or, tout comme dans «Daffodils » (22), quand le jaune et la lumière surgissent à nouveau dans l'écriture, ils n'ont plus la même portée. Que reste-il de cette expérience transperçante, de la lumière qui «traverse » et «irradie " («coming through", «irradiating», 22) ? Quel est ce jaune qui «se multiplie à l'infini» mais n'est plus le même (« it multiplies / Endlessly. But nothing is the same », 22) ? La lumière et le jaune peuvent-ils s'apparenter à des signes d'espoir, même ténus, comme la lumière crépusculaire dans « Little Jerusalem » (10-12) ? Ne sont-ils plus que traces, vestiges d'une grâce et d'une direction perdues dans un monde qui ne fait plus sens?

\section{Du jaune infini à ses versions dénaturées et à la lumière obscurcie : paradis artificiels et traces fracturées d'une grâce disparue}

Dans « Citrus », le jaune se fait infini au milieu du gris et de l'hiver :

All lemons are green to begin with, all oranges.

Ours, the nurslings of grey Irish light,

Turn yellow in winter. Meanwhile, through October, 
In the tall windows, higher than human height, Incarnate joy, against the logic of seasons, Goes on ripening. Slowly a solstice approaches,

Drenched in cold moonshine, when the little tree Self-pollinating, like a private mystery

Kept behind glass, from a collapsing world,

Goes south in itself, beyond poverty and death, To infinite yellowness....

They are selling it

Already, on the Sicilian squares,

For less than nothing, as the sun moves up

Through the latitudes now, to catch us unawares

At the back end of January, still waiting,

In the days that never rise above themselves,

To slice it for its zest, against the grain

Of whisky, the false lift of gin and tonic. (WS, 18) que les cheveux blonds de la serveuse du pub ou la couleur ambrée du whisky ( blonde hair ", «the colour of Jameson ", WS, 52). Le blond roussi par le feu peut être perçu comme un affaiblissement, une version dénaturée des auréoles dorées, des mandorles des êtres en grâce. L'ambré du whisky s'apparente lui aussi à un obscurcissement. Il crée l'illusion d'un état de gloire, bien éloigné de celui des êtres ou du Christ en gloire dans l'iconographie religieuse: «Bathed in their own reflected glory, / Men are drinking» (52). Nous ne sommes plus dans le temps circulaire du mythe qui s'oppose à la linéarité de l'histoire ; la seule forme de circularité restante est le cycle des saisons sans qu'il induise pour autant une idée de recommencement, de régénération ${ }^{16}$. La «roue des saisons » (« the wheel of seasons », 52) ne renvoie pas à un rythme cosmique harmonieux mais à une roue de la Fortune déréglée. Le pub brûlé pour des questions d'assurance devient l'emblème d'un monde calciné, en ruines, où même l'histoire ne fait plus sens puisqu'il n'y a plus de chemin à suivre si ce n'est l'inconnu : « the unforseeable country ahead $»(52)^{17}$.

De même, dans «Dying Generations », la linéarité du temps est brisée et seul reste l'instant présent: «On such a day, with the future cancelled out,/ We live in the 
present " (WS, 35). Le titre choisi renvoie au poème de Yeats "Sailing to Byzantium ", qu'il déconstruit ("Those dying generations», Yeats, 193). Par le truchement de la répétition du motif de la chute - l'individu tombant d'un arbre répondant à l'oiseau à terre («An odd bird down", « somebody felling a tree», WS, 35) - il expose un monde postlapsaire dans un présent privé de futur et de passé où seule la Chute se rejoue. Si un retour à une linéarité de l'histoire qui ferait sens est annoncé, cela reste de l'ordre de la prédiction, et la seule certitude réside dans le quotidien ${ }^{18}$ :

[...] Somebody found

An odd bird down the river

Where Desmond opened a path, between his fields

of wheat and potato.

On such a day

Who wouldn't feel, when the mist is everywhere,

Serotonin drops, of mood into light,

Are the one solution? [...] I sense the oncoming fronts

From the Atlantic, days before they reach us

Deep inland. A great ache

Or is it change, or the slow beginning

of something inherited, as the old man

Goes to pieces? I no longer believe in anything

But the greenness, the greyness,

The eternal everydayness

of Ireland. Time, they tell me, will come back -

The past, the future. Meanwhile I wake

With an empty mind, to a high ceiling,

A jug of clear spring water,

The buzz of a chainsaw, somebody felling a tree. (35)

La répétition produit une forme d'abolition du temps profane sans pour autant générer une projection dans le temps du mythe. Le présent gris et vert, ordinaire et brumeux, seul point d'ancrage restant, se fait à la fois croyance séculière et temps de dislocation, comme l'illustre l'image du vieil homme qui se désagrège. La décrépitude de ce dernier contraste avec l'absence de vieillissement dans le monde hors du temps de "Sailing to Byzantium » ("That is no country for old men », " the artifice of eternity ", 193). De même, l'oiseau à terre de Clifton s'oppose aux oiseaux chantant dans les arbres de Yeats, ainsi qu'à l'oiseau doré, mythique, à savoir la forme transcendée dans laquelle le poète se projette (« birds in the trees ", " set upon a golden bough to sing ", 194). Ici, la fracture du présent est aussi figurée par la césure du poème au début du treizième vers qui le scinde en deux parties séparées par un blanc. Dans ce quotidien sans espoir et qui se répète, l'unique échappatoire est une lumière en bouteille : le paradis artificiel des gouttes de sérotonine. Si le poème de Yeats s'achève sur un au-delà enchanté où l'or prédomine, où l'œuvre d'art donne l'espoir d'une éternité (Yeats, 194), dans celui de Clifton, il n'y a que l'immanence.

Si cet «ici et maintenant» n'est souvent qu'un quotidien gris, s'il n'est que momentanément nouvel Éden ou vraie Jérusalem séculiers ${ }^{19}$, il n'est pas pour autant dépourvu de lumière. Dans " Marriage », tout comme dans "Little Jerusalem », la lumière est liée à l'amour. "Marriage " peut être lu comme une allégorie de la vie d'un couple marié, où les figures féminines se juxtaposent, se substituent au fur et à mesure que le poème retrace la vision que les deux membres d'un couple ont l'un de l'autre au fil des années communes. Il peut aussi être défini comme une ekphrasis de la photographie choisie pour la couverture du recueil. De ce fait, il entre en résonance avec l'univers de Samuel Beckett ${ }^{20}$. En effet, ce cliché est la version miroir de la photographie de David 
Davison intitulée Father and Son on a Mountain Road to Nowhere, représentant un homme et un enfant marchant dans la neige au milieu des montagnes de Dublin. Cette image a été publiée la première fois pour illustrer les souvenirs d'enfance de Beckett avec son père ainsi qu'un extrait de son ouvrage Worstward Ho. Cet écho beckettien est renforcé par l'ajout en exergue du poème d'un extrait de la citation de Worstward Ho dont l'image se faisait initialement l'illustration ${ }^{21}$. Le corps du poème renvoie aussi à la novella de Beckett de par la langue elliptique, les répétitions avec variations et les personnages du vieil homme et de l'enfant qui se superposent et se substituent l'un à l'autre. Cependant, si la vision de l'homme et de sa fille dans la première et la dernière strophe semble être celle de la photographie de couverture, elle est perçue avec un plus grand effet d'éloignement :

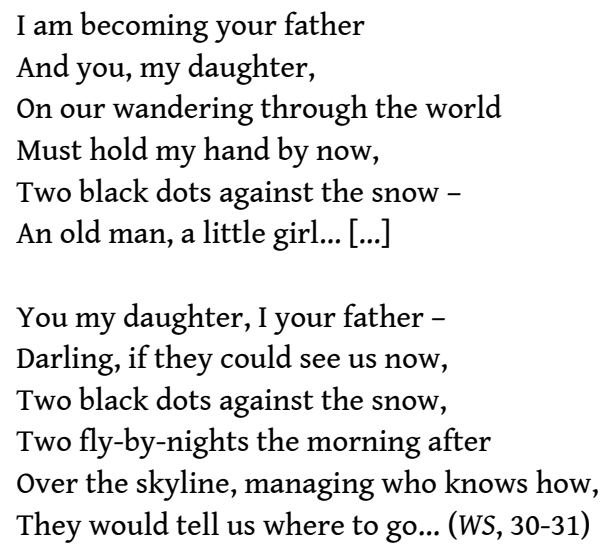

Cette répétition avec variation de la première à la dernière strophe crée de surcroît un « effet de cadrage » qui ajoute au « degré de picturalisation du texte », pour reprendre la formule de Liliane Louvel (Louvel 33, 42). Dans « Marriage ", c'est une «étrange lueur » («A strange glow») qui annonce l'aube de l'amour, «étrange lueur» qui fait écho aux êtres étrangement éclairés (" strangely lit ») après l'amour dans " Little Jerusalem » (WS, $30,11)$. Cette lueur, qui annonce le commencement, se transforme en vraie lumière au moment de la rencontre et de la naissance de l'amour ("And then the light », 30). Or, le parcours commun au sein de la vie est décrit comme une traversée de paysages enneigés auxquels le poète se réfère par le néologisme "snowscapes » et par le terme de " désert ", d'« étendue sauvage » (« wilderness ») qui renvoie intertextuellement à la traversée du désert du Christ. Comme le souligne le choix de la citation de Worstward Ho, ce parcours n'a rien de l'exaltation du voyage de Westward Ho, roman historique britannique à la philosophie impérialiste de Charles Kingsley dont Beckett détourne le titre. Il n'y a pas pour autant «Cap au pire » pour reprendre la traduction officielle du titre de la novella de Beckett ; il n'y a aucun cap dans le texte de Clifton, seulement « une route qui ne mène nulle part », comme la fin du titre de la photographie de Davison le suggérait (« a [...] road to nowhere »). Il n'y a aucune direction à suivre, malgré le souhait d'en trouver une : "Another / With clouded specs, directionless, / You lean on me, as we stagger on ». Par cette image des lunettes qui n'offrent qu'une vision obscurcie, Clifton évoque et déconstruit la première épître aux Corinthiens selon laquelle nous ne percevons pas la totalité des choses ici-bas : "for now we see through a glass, darkly (Corinthians I, 13 : 12). Or, ici, il n'a pas l'espoir de connaître le visage de Dieu. Dans ce monde privé de Dieu et d'au-delà, où le sens n'est pas à chercher ailleurs mais ici-bas, où les hommes semblent condamnés à l'errance, il reste la présence de l'un à l'autre. Cet amour, même affaibli, presque plus lumineux, est le rempart qui permet de ne pas chuter. À un niveau intratextuel, cela fait écho à « Exiles ", où la vie n'est qu'errance et aliénation et où seul 
l'amour fait sens, même si, dans ce poème antérieur, les notions de sens et de but n'échappent pas totalement : «And I say to you "Let us make a home / In ourselves, in each other..." [...] love, that throws us together / In the back seat of our own destiny / Where one dreams, and the other gives directions » (Clifton 2014, 71). C'est la fin de ce poème que Clifton cite sans le nommer dans l'article «Coming Home» pour illustrer le fait que, dans un monde semblable à un carrefour où les hommes sont en transit permanent, la seule vraie maison n'est pas un point fixe, localisable; le seul sentiment d'appartenance provient de la présence de l'être aimé doublée de l'utilisation de sa langue natale : « As for home, we carried it inside us, a marriage and a mother-tongue. The poet was a citizen not of place but of language » $(2006,11)$.

Enfin, le questionnement qui s'opère dans "The Approaches » offre un bilan existentiel qui peut être perçu comme un résumé de la quête de sens qui s'exprime tout au long du recueil :

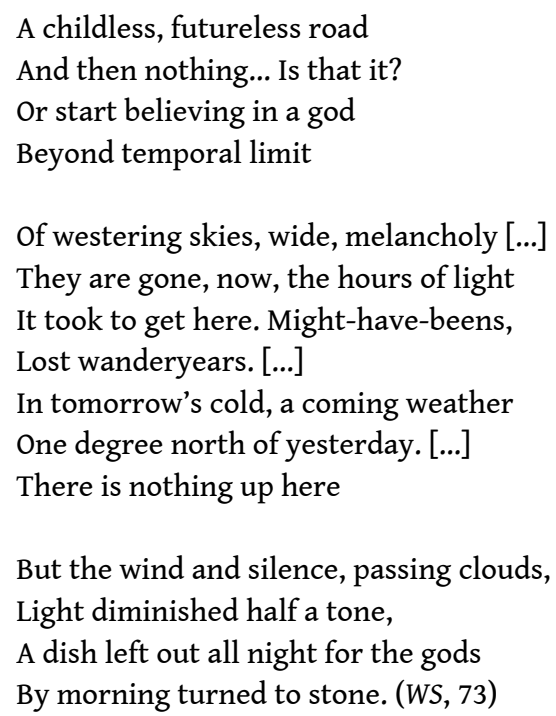

Que reste-t-il dans un monde où les dieux se sont retirés et où il n'y a plus de futur? Le sens n'est pas à trouver à l'extérieur, dans la croyance en un Dieu, mais dans la présence du monde et au monde, dans ce qui perdure. En effet, même si la lumière éclatante a disparu et si elle n'est plus qu'une trace affaiblie, elle est cependant encore présente dans ce monde réduit à des ruines, des restes, dont ceux pétrifiés des offrandes aux dieux.

Ainsi, Harry Clifton nous dépeint un monde en ruines et gris, sous la pluie et la brume, dans lequel l'histoire ne fait plus sens, un hiver éternel que les hommes, exilés, traversent sans fin, sans but. Ce quotidien sombre, qui se répète, peut être assimilé à une longue nuit, à l'hibernation auquel le titre du recueil fait référence. Pourtant, pour reprendre les vers inauguraux de "Pipistrelle», la lumière n'est jamais totalement absente de ce monde : "At no point, in the whole of that northern night, / Was there total eclipse of light »(WS, 65). C'est d'ailleurs bien souvent autour de l'écriture du jaune et de la lumière, qu'elle soit éclatante, pure, affaiblie, obscurcie, ou factice, que le sens se construit. C'est aussi par les traversées intermédiales, intratextuelles et intertextuelles. Elles créent un dialogue fécond au centre du questionnement sur l'avoir-été, l'être et le devenir qui s'exprime au long du recueil. Ces traversées s'effectuent dans les deux sens puisque les sous-images et les sous-textes sont à la fois le point de départ du cheminement des mots, ce qui parcourt le texte, et ce qui est déconstruit, transformé. Enfin, dans la quête de sens qui s'écrit au fil des pages, le jaune et la lumière, même dénaturés, sont à la fois la trace 
de ce qui a été perdu et de ce qui reste - paradis artificiels ou Éden séculiers, clarté de l'amour ou d'un effet de présence, d'une communion avec le monde, dans l'être, ici et maintenant. Cependant, dans ce monde, ce jaune et cette lumière sont fragiles et momentanés. Ils semblent condamnés au morcellement et à l'altération.

\section{BIBLIOGRAPHIE}

Aquien, Pascal. «W.H. Auden, de l'Atlantide à la Nouvelle Jérusalem ». Études anglaises 54 (2001) : 41-54. www.cairn.info/revue-etudes-anglaises-2001-1-page-41.htm (dernière consultation juin 2016).

Auden, W.H. Collected Poems (1976). London: Faber \& Faber, 1991.

Auden, W.H. « Dingley Dell \& The Fleet ». In The Dyer's Hand and Other Essays. London: Faber \& Faber, 1963, 407-28.

Beckett, Samuel. Worstward Ho. New York City: Grove Press, 1984.

Clifton, Harry. «Coming Home ». Irish Pages. The Home Place. Ed. Chris Agee. Vol. 3, No 2. Belfast: Nicholson \& Bass, 2006, 7-16.

Clifton, Harry. Secular Eden, Paris Notebooks 1994-2004. Winston-Salem: Wake Forest UP, 2007.

Clifton, Harry. The Holding Centre, Selected Poems 1974-2004. Northumberland: Bloodaxe Books, 2014.

Clifton, Harry. The Winter Sleep of Captain Lemass. Northumberland: Bloodaxe Books, 2012.

Durand, Gilbert. Les Structures anthropologiques de l'imaginaire. Paris : Dunod, 1969.

Eliade, Mircea. Le Mythe de l'éternel retour (1969). Saint-Amand : Gallimard, 2014.

Eliot, T.S. Collected Poems, 1909-1962 (1974). London: Faber \& Faber, 2002.

Heaney, Seamus. New Selected Poems, 1988-2013. London: Faber \& Faber, 2015.

Joyce, James. Ulysses (1922). London: Penguin Books, 2000.

Keatinge, Benjamin. « Home to Original Homelessness, review of The Winter Sleep of Captain Lemass by Harry Clifton, World Without Maps by Geraldine Mitchell, and Happy Hour by Andrew Jamison ». Poetry Ireland Review 109 (Spring 2013), ed. John F. Deane: 101-104.

Louvel, Liliane. Texte/image, Images à lire, textes à voir. Rennes : PUR, 2002.

Marin, Louis. Des pouvoirs de l'image, gloses. Paris : Éditions du Seuil, 1998.

Nancy, Jean-Luc. The Birth to Presence. Trad. Brian Holmes. Stanford: Stanford UP, 1999.

O'Brien, Eoin. The Beckett Country: Samuel Beckett's Ireland. Dublin: The Black Cat Press Limited in association with London: Faber \& Faber, 1986.

O'Toole, Fintan. « When TV went to the wire and "Seldom Seen Kid" was often heard ». The Irish Times (20 décembre 2008). http://www.irishtimes.com/news/when-tv-went-to-the-wire-andseldom-seen-kid-was-often-heard-1.1275326 (dernière consultation octobre 2015).

Wordsworth, William. Selections from William Wordsworth. Poetry and Prose. London: Methuen, 1966. 
Yeats, W.B. The Collected Poems of W.B. Yeats. Ed. Richard J. Finneran. Revised $2^{\text {nd }}$ ed. New York: Scribner, 1996.

\section{NOTES}

1. Académie du Arts Council irlandais qui rassemble les artistes ayant apporté une contribution remarquable aux arts irlandais dans le domaine de la littérature, de la musique ou des arts plastiques.

2. Toutes les traductions de citations de Clifton sont les miennes.

3. Voir par exemple «The Winter Sleep of Captain Lemass » ou «The Approaches » (WS, 44-46, 73).

4. Dans «Coming Home» (2006), Clifton retrace l'évolution de ces deux Irlande. Ce pays divisé, décrit d'abord comme lugubre ("the bleak futurelessness of two Irelands", "grey republic, bloody north », 7, 9) devient un carrefour (« a landing-strip », « a global intersection ») marqué à la fois par une dimension cosmopolite, décadente, et isolationniste, voire sectaire, au nord (10, 12).

5. Pour transposer l'image de «Coming Home» (Clifton 2006, 15).

6. Nous transposons ici à l'intertextualité la pensée de Louis Marin à propos des liens entre texte et image : «La "traversée" peut, je crois, se dire dans les deux sens, selon les deux directions, l'image traverse les textes et les change ; traversés par elle, les textes la transforment » (9).

7. Auteur qui, comme le rappelle Clifton, a vécu dans cette région d'Irlande (WS, 25).

8. Bloom est censé être né au 52 Upper Clanbrassil Street, à Little Jerusalem qui est l'ancien quartier juif de Portobello à Dublin où vit Clifton (voir Keatinge, 102). À un niveau intratextuel, ceci renvoie à «Benjamin Fondane Departs from the East» (Clifton 2007, 200) : «[...] all of us transmigrating / Like souls, through the neutral space on the map./ Athens and Jerusalem, Ulysses and the Wandering Jew - There we all go [...]». Au sein de WS, l'exil s'écrit également en écho avec l'Odyssée par l'expression « Grecs-Irlandais » ("Irish Greeks ») dans "The Year of the Yellow Meal » (41).

9. Dans The Burning Perch, le gris est aussi celui d'un présent sans passé ni futur dans «The Grey Ones » ou celui d'un monde et d'êtres sans espoir de salut dans «Greyness is All » (Macneice, 12-13, 32).

10. À un niveau intratextuel, ceci fait écho au poème "After Ireland »: "And the notion of heaven, / of retribution, / Melting, like exhaust fumes, / In desecrated space. / To be on earth, / In Ireland. [...] Selfconsciousness / Without God. A solitude, / A self-sufficiency / Feeding not on roots, / But on the dream of roots » (Clifton 2014, 133-134).

11. L'Arcadien est tourné vers un Paradis perdu, un Éden d'avant la chute et la souffrance, et l'Utopiste vers un bonheur futur. Voir l'essai d'Auden « Dingley Dell \& The Fleet » $(1963,409)$. De plus, à propos de l'Arcadien, de l'Utopiste et de l'alternative, à savoir « un autre espace de bonheur retrouvé : celui de la poésie dont l'un des fondements conceptuels est la théorisation de la Pentecôte comme don des langues ", voir Aquien, 50-54.

12. Par rapport à la vision chrétienne de l'histoire comme « une ligne droite », un temps qui « est réel parce qu'il a un sens ", voir Eliade, 160-161.

13. À un niveau intratextuel, ce lien entre amour et lumière fait écho à "Vaucluse ", poème originellement publié dans The Liberal Cage (1988) : "Cognac, like a gold sun / Blazed in me, turning / The landscape inside out [...] and I saw again / France, like a blue afternoon / Geniuses make hay in, and drink improves - / The work fields, the yellow sheaves / In shockwaves, perceived / And lit from within, by love. [...] Transfigured, / Not yet fallen from grace / I saw us, / not as we are / But new in love, in the hallowed place / Of sources, the sacred fountains of Petrarch and René Char » (Clifton, 2014, 65-66). Si dans « Vaucluse » le poète établit un lien entre 
amour, lumière, grâce et sacré, ces visions positives n'existent que de manière artificielle : d'abord sous l'effet de l'alcool, puis de la réinvention poétique (deux paradis artificiels). L'obscurcissement marque un retour à la réalité qui est, elle, grise et sombre: «the train / Occluded in grey rain / Above Lyons, and the Rhône valley / Darkened » (Clifton, 2014, 66).

14. En écho à «Little Jerusalem » et par extension au poème de W.H. Auden « Vespers » (1976, 637-639), dans "Christiana », l'être est défini comme la nouvelle Jérusalem, et donc semble-t-il, comme la manière d'accéder à une forme de félicité dans un monde dystopique. Cette nouvelle Jérusalem est séculière, ici et maintenant, et non religieuse : «For an instant, poised above time, / Being, not doing, is the real Jerusalem - [...] / Dystopia, where the Lords of Misrule, [...] / Catch holy innocents, and drag them off to school. / They say whoever leaves you, Christiania, dies - / The laidback, the naïve, / Their hair down to their feet, like Adam and Eve, / Speaking if not in tongues, at least in Danish, / Dreaming other Edens as they vanish » (WS, 78).

15. Par l'opposition entre sud lumineux et nord gris, et par la vision de l'alcool qui permet d'accéder à une sorte de paradis artificiel, ce poème fait lui aussi écho à "Vaucluse " (Clifton 2014, 65-66). Voir supra note 9 pour le lien entre « Vaucluse » et « Little Jerusalem ». L'insistance sur l'opposition entre sud et nord, gris et jaune, traverse l'œuvre de Clifton. Dans WS, ce thème est par exemple repris dans "False Meridian », où le nord, associé au quotidien, s'oppose au sud qui serait lié au temps du mythe ("North to South, between two times, / The daily, the eternal », 64).

16. Par rapport au mythe, au schème rythmique du cycle, à la circularité calendaire et à la répétition qui s'oppose au "temps concret historique ", "continu » voir Durand, 322-325 et Eliade, 11-12, 50-51, 181-182.

17. Nous retrouvons de manière similaire l'expression d'un lieu « abandonné par l'histoire » qui se fait lieu «d'exil» dans «Letters from Buenos Aires »: «abandoned by history, / A city of exile» (WS, 83).

18. Ce futur semble venir de, et être défini par les États-Unis. Nous retrouvons ici une allusion au lien entre l'Irlande et les États-Unis exprimé de manière ironique dans «Little Jerusalem », WS, 11 : «A wandering Jew, in a faraway place / That is not America / That must be some mistake ». Par rapport à ce lien, voir aussi « The Year of the Yellow Meal» (WS, 39-41) ou « Coming Home » (Clifton 2016, 7-16).

19. Voir « Daffodils » ou « Christiania », WS, 22, 78.

20. Keatinge note de surcroît un lien entre Beckett et Clifton dans le poème « The Winter Sleep of Captain Lemass ", en soulignant la référence que Beckett fait à l'exécution de Noel Lemass dans son roman Mercier et Camier, et souligne un lien possible entre l'œuvre de Beckett et le sentiment d'aliénation qui parcourt le recueil («Clifton perhaps leans on Beckett to convey his sense of "original homelessness" which pervades the volume ", 102).

21. La photographie de Davison a été initialement publiée dans l'ouvrage de Eoin O'Brien intitulé The Beckett Country: Samuel Beckett's Ireland et créé pour célébrer le quatre-vingtième anniversaire de Samuel Beckett en 1986 (Chapitre 2, « The Dublin Mountains », 54). Cette page est accessible sur le site de la UCD Digital Library: http://digital.ucd.ie/view/ivrla:6557 (dernière consultation octobre 2015). 


\section{RÉSUMÉS}

Le mouvement et le déplacement sont au cœur de l'œuvre de Harry Clifton. Le recueil intitulé The Winter Sleep of Captain Lemass est ainsi placé sous le signe de traversées : dans l'espace, le temps, la littérature, mais aussi d'un medium à l'autre (de l'image au texte et réciproquement). Cependant, dans le monde que décrit le poète irlandais contemporain - un monde privé de Dieu, condamné à la répétition du présent, où le passé est un espace enneigé qui enlise, pétrifie et où la possibilité d'un futur semble abolie - ces déplacements peuvent tourner court, et aboutir à une forme de stagnation, à une errance sans but. Cet article se propose d'étudier plus précisément l'écriture du jaune et de la lumière, ainsi que le fonctionnement de l'intermédialité, de l'intratextualité et de l'intertextualité. Si le jaune et la lumière peuvent être artificiels ou s'obscurcir, n'être plus que des traces - signes et restes - d'un sens perdu et d'une grâce disparue, ils percent la grisaille de l'ordinaire et du quotidien. Ils peuvent aussi être le symbole d'un effet de présence et/ou de l'amour, de moments où l'être est transpercé et redéfini. Enfin, au cœur de l'écriture même, la traversée intertextuelle est à double sens. D'un côté, les sous-textes qui parcourent l'espace poétique sont le point de départ du cheminement des mots; ils servent de fondation à l'expression d'un questionnement sur l'avoir-été, l'être et le devenir. De l'autre, ils sont ce que le poète déconstruit et transforme, leur donnant un nouveau tour. Ceci crée de multiples effets de résonance qui se font dialogue fécond d'un texte à l'autre, et qui s'étendent en se complexifiant de manière intermédiale et intratextuelle.

Movement and displacement are at the heart of Harry Clifton's work, and The Winter Sleep of Captain Lemass exhibits multiple forms of crossings: through space, time, literature, or media (from image to text and vice versa). Nonetheless, in the world described by this contemporary Irish poet-a world without God, where the present repeats itself, where the past is perceived as a snowy, freezing, petrifying place, and where the future seems to have been cancelled-these movements can become forms of aimless wandering, of stagnation, or be defined as the way to nowhere. This article focuses on the representation of yellow and light and on how intermediality, intratextuality and intertextuality operate. Yellow and light can be artificial, altered or darkened, they can appear as traces, as what remains of a lost direction, meaning, and grace, but they still break through and penetrate the greyness of ordinary life. They can also represent moments when love or presence is experienced so deeply that they affect the self, going through it, to its core, and redefining it. Finally, this essay will examine how intertextuality can be considered as a two-way crossing. Throughout the book, quotations and references are a starting point leading to a new written path; they work as foundations, as what sustains the poet's questioning about what was, is and will be. They are also what the poet deconstructs, transforms and rewrites, hence giving them a new meaning and direction. As a result, effects of resonance create a fruitful dialogue between texts, and reverberate through intermediality and intratextuality. 
INDEX

oeuvrecitee Winter Sleep of Captain Lemass (The)

Keywords : yellow, light, present, intertextuality, crossing

Mots-clés : jaune, lumière, présent, intertextualité, traversée

\section{AUTEURS}

\section{CATHY ROCHE-LIGER}

Cathy Roche-Liger est l'auteur de la première thèse française sur le poète irlandais Paul Durcan (« Poétique du pictural dans l'œuvre de Paul Durcan », 2011). Rattachée à l'équipe B1 du FoReLL de l'université de Poitiers, elle a écrit plusieurs articles sur P. Durcan, notamment sur ses ekphraseis, et également travaillé sur les poètes W.H. Auden, Michael Longley, John Montague, Harry Clifton, Trevor Joyce et Geoffrey Squires. Elle s'intéresse particulièrement à la poésie irlandaise contemporaine et aux interactions entre le visuel et le verbal. 